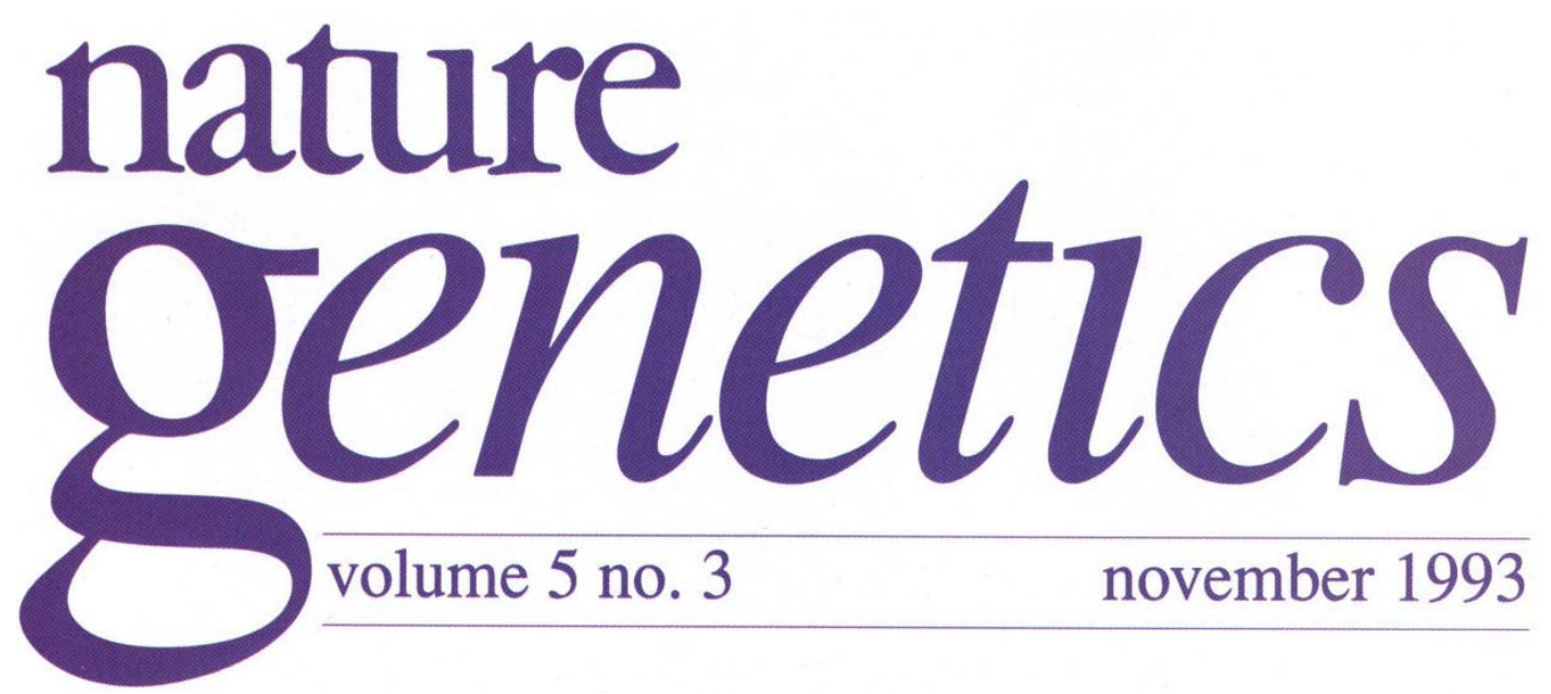

\title{
Human genetics at the Whitehead
}

Given the considerable expertise in mammalian genetics among the ranks of the Whitehead Institute for Biomedical Research in Cambridge, Massachusetts, it is perhaps a little surprising that it took more than a decade for the organizers of its annual symposium to consider human genetics worthy of its undivided attention. At the eleventh annual Whitehead symposium*, there was no shortage of talks pointing out the many advantages of studying model organisms to gain insight into the structure and function of the human genome. But as Gerald Fink, director of the Whitehead (and a yeast geneticist), affirmed, human genetics is no longer the poor relation in the field of genetics, a fact that the Whitehead symposium was now confirming.

The past few years have seen considerable progress in human genome research, notably with the development of a 2-5 centiMorgan (cM) genetic map and the successful isolation of many elusive disease genes, such as Huntington's disease (HD) (F. Collins, National Institutes of Health). What is missing, however, are the advanced technologies that will be vital for accelerating the pace of DNA sequence collection and interpretation. HD provides a sobering example: the gene sequence has offered negligible insight (so far) into its normal function, and no immediate clues have emerged from the study of its tissue expression pattern (see Strong et al., pages 259-265). D. Botstein (Stanford University) pointed out that sequence alone is not akin to knowledge. The sequence of the Saccharomyces cerevisiae genome,

*'Human Genetics', Eleventh Whitehead Institute Symposium, Cambridge, Massachusetts, USA, October 3-5, 1993. less than $1 / 200$ th the size of the human genome, should be complete within the next four years, and, coupled with the numerous experimental advantages of yeast (such as complementation analysis), should prove invaluable. Similar hopes are being pinned on the sequence of the Caenorhabditis elegans genome (R. Waterston, University of Washington).

Complex traits: Perhaps the biggest challenge facing human genetics is to extrapolate success in understanding monogenic disease to more complex disorders, such as diabetes, obesity and cancer. Progress will often hinge on the development and study of suitable animal models, and is being facilitated by the mouse genetic map, which currently consists of more than 2,000 markers spaced on average less than $1 \mathrm{cM}$ apart (E. Lander, Whitehead Institute). A growing trend in analysing animal crosses is towards the automation of microsatellite typing (by adapting DNA sequencing technology), which is both highly efficient and accurate, and assisting the mapping of new susceptibility loci for type 1 diabetes (J. Todd, University of Oxford). Hereditary factors are thought to have a greater relative role in obesity than in many other complex traits (J. Friedman, Rockefeller University). One of the known loci in mice, $o b$, is a putative loss-of-function mutation in which homozygous mice eat more and exercise less than is normal. When these mice are sutured to wild-type animals, they lose weight, suggesting they are indeed missing an essential regulatory factor. The defective oblocus has been confined to some 300 kilobases on chromosome 6 , and candidate genes are under scrutiny. A rather 
different definition of complexity applies to the so-called prion diseases, but, as described by C. Weissmann (University of Zürich), mouse mutant studies are again proving invaluable. Homozygous mice lacking the prion gene are still resistant to scrapie two years after infection. Interestingly, mice with hamster prion transgenes are susceptible to hamster prions but resistant to murine prions, suggesting that species homology is important, perhaps in provoking a conformational change to produce an infectious variant. There was no better illustration of the exquisite sensitivity of phototransduction than the remarkable 'back-ofthe-envelope' calculation of J. Nathans (Johns Hopkins School of Medicine) showing that the potential energy expended in dropping a small coin one millimetre would translate into a discernible flash of light for the entire world's population.

Cancer is a complex genetic disease for which mouse studies have again come to the fore (D. Housman, Massachusetts Institute of Technology; N. Hastie, MRC Human Genetics Unit, Edinburgh). Homozygous male mice lacking $\alpha$ inhibin develop bilateral testicular (and later adrenal) tumours, suggesting the existence of a novel signal transduction cascade involved in gonadal tumorigenesis (A. Bradley, Baylor College of Medicine). A growing number of hereditary cancer genes have been identified, including the gene for multiple endocrine neoplasia 2A (MEN2A), which B. Ponder (University of Cambridge) and colleagues have shown to be the RET oncogene. Ponder, "a slender, soft-spoken man with kind eyes" according to a recent magazine article (Self, October 1993, p. 166), described how certain RET mutations, even specific substitutions at a given codon, are associated with a given phenotype. Whether RET mutations also give rise to MEN2B or Hirschprung's disease, which was recently mapped to the same region of chromosome 10, remains to be seen, as does the identity of the hereditary breast and ovarian cancer gene (M-C. King, University of California, Berkeley).

Cancer development has been linked to the relaxation of imprinting, or parental allele-specific expression, which has been described for four mouse genes. D. Gottschling (University of Chicago) has analysed the silencing of genes at the telomere of yeast chromosomes - a phenomenon that may have significance for human disorders.
The inactivation of URA3, for example, depends on both its proximity to the telomere and the presence of other loci. One of these, SIR3, is required for the basal silencing of genes, and, if overexpressed, is lethal to the cell. SIR 3 may be the equivalent of mammalian MeCP proteins (A. Bird, University of Edinburgh). These nuclear proteins bind naturally to $\mathrm{CpG}$ dinucleotides and are thought to be important in controlling gene expression. Experiments are under way to mutate these genes in mice, which will allow comparison of the role of these proteins with DNA methyltransferase (R. Jaenisch, Whitehead Institute). S. Tilghman (Princeton University) has focused on the expression of a physically linked pair of imprinted genes, $H 19$ and Igf-2, which are expressed from the maternal and paternal chromosome respectively. These genes share common downstream enhancer factors, although whether they compete for such regulatory molecules is unclear. X-chromosome inactivation is associated with the expression of the XIST gene from the inactive X chromosome (M. Lyon, MRC Radiobiology Unit, Didcot). The XIST transcript is associated with the silent X (J.B. Lawrence, University of Massachusetts Medical Center), but its molecular mode of action remains a puzzle.

While new technology is urgently required in the sequencing realm, new methods are being developed to solve other 'ornery aspects' of human genetics, from the study of meiotic events in sperm (N. Arnheim, University of Southern California) to the application of deletion maps and classical genetics for pinpointing genes involved in sex determination (D. Page, Whitehead Institute). A technique called representational difference analysis allows the isolation of a DNA sequence that is unique between two otherwise identical samples (M. Wigler, Cold Spring Harbor Laboratory). The method has already been applied to pulling out probes at several mouse loci using inbred strains, and although more difficult, may prove useful in isolating human probes corresponding to hemizygous or homozygous deletions in tumours, as well as other types of rearrangement.

\section{Money matters}

In last month's editorial (Vol. 5, 101-102; 1993), the US Army was said to have received a $\$ 210$ appropriation for breast cancer research. This should, of course, have read $\$ 210$ million. 